\title{
Interactive comment on "Biotic and abiotic transformation of amino acids in cloud water: Experimental studies and atmospheric implications" by Saly Jaber et al.
}

\section{Anonymous Referee \#2}

Received and published: 25 August 2020

In this work the authors conducted microcosm experiments with the aim of differentiating the roles of biotic and abiotic transformation of free amino acids in cloud water. In the experiment, they utilized 19 types of amino acids, four bacterial strains or photo-bioreactors, attempting to mimic ambient cloud conditions. With the kinetic loss/production data of amino acids, they concluded that previous studies may have overestimated the abiotic degradation rates of amino acids, and future modeling efforts should take the biotic and abiotic transformation of amino acids into account. Overall, I think the authors did a solid job in terms of writing and offered an interesting dataset in amino acid dynamics in the field of cloud chemistry. The data seem to be sound. However, I have some concerns the authors need to address before publication. 
The authors claimed that they developed a new analytical technique that can analyze amino acids, without the need of any preparation such as derivatization, using UPLCHRMS. This is nice effort, but I am surprised that the incubation medium they used, containing $\mathrm{mM}$ of ions such as $\mathrm{Na}, \mathrm{Ca}$ an $\mathrm{K}$ etc. (Table $\mathrm{S} 1$ ), which would directly impact ion source and ionization process, was directly injected to the LC-MS. I don't think any mass spectrometry expert would be happy if you inject such a solution to the instrument. I am wondering what's the inject volume they used and how they can maintain a consistent sensitivity with such high ion strength solution (or how long). In addition, external calibration curves were used to quantify the amino acids in cloud water medium. Did you use the same water medium for the standards? If not, this might be a problem with the matrix effect. It may be also helpful to show the LC-MS chromatograms in the supplementary section. The bottom line is that more info is needed for this new approach you developed.

I like the experimental approach, such as clearly separating abiotic and biotic factors, and using free amino acids and single bacterial strains, which allowed you to tease out the convoluted factors observed in field samples. However, the authors need to realize/justify their experimental conditions which I think are far away from those of the field, thus more discussion is needed. For example, they used $1 \mathrm{uM}$ of 19 types of amino acids, which represent $19 \mathrm{uM}$ amino acids or $684 \mathrm{ug} \mathrm{C/L}$ (assuming $3 \mathrm{C}$ per amino acid); in contrast, the cloud water only contained 2.4-74.3 ug $\mathrm{C} / \mathrm{L}$, cited from the Introduction of the manuscript. Your rate calculation is dependent on the concentration, thus the extremely high concentrations you used could have led to a conclusion not relevant to the field (the rate constant could also change depend on how the bacteria take up the substrate). In addition, when bacteria are just harvested from culture medium, in a way they have been pre-trained to take up labile organic matter rapidly, thus the biotic loss rate you obtained could have been overestimated. Bacteria in the cloud water, on the contrary, may not be that active often due to substrate limitation. Similarly, the abiotic transformation rates would be different when you have an organic matrix present, like cloud water. As mentioned in the Introduction, organic matter in the cloud water is

Printer-friendly version

Discussion paper 
complicated, including many different compounds, which may include quenchers and photosensitizers, the rates you obtained may not represent those of field. I think all these need to be factored in when you try to argue against previous studies, or apply these data to the field. I would like to see more discussion along these perspectives.

Many places in the Introduction, there are too many references which kind of stops the flow. I would suggest you only choose the key ones.

Page 3 line 28: "biotranform"? You meant: ". . shown to biotransform. .." Page 7 line 25: no need to list all these amino acids here. Page 8 line 3: should be "due to" Page 8 line 7: should be "bacterial strain" Page 8 line 9: I think "production" is a better word than "synthesis" here. Page 9 line 26: delete "in the experiments", redundant Page 11 line 5: delete the "..." Page 11 lines 10\&17: the equations did not show up right. It might have something to do with the formatting. Page 12 line 8: delete "It is obvious that" Page 12 line 30: right, but as I mentioned before, your experimental conditions may not be that relevant, either.

Interactive comment on Biogeosciences Discuss., https://doi.org/10.5194/bg-2020-250, 2020. 